\title{
Four COVID-19 Cases of New Variant B.1.351 First Emerging in South Africa in Chinese Passengers on Same Flight - Shenzhen, China, January 2021
}

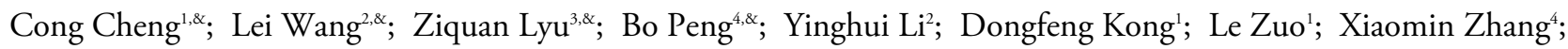

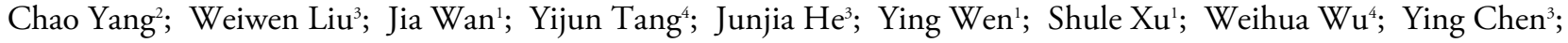
Guangnan $\mathrm{Liu}^{3}$; Junjie Xia ${ }^{5}$; Xuan Zou ${ }^{5}$; Renli Zhang ${ }^{4}$; Qinghua $\mathrm{Hu}^{2, *}$; Shujiang Mei ${ }^{1, *}$; Tiejian Feng ${ }^{5, t}$

At 04:50 on January 1, 2021, a 36-year-old Chinese project manager (Case A), a 29-year-old Chinese worker (Case B), and a 53-year-old Chinese businessman (Case C) returned from Africa (Case A and B from South Africa and Case C from Lesotho) on the same flight and tested coronavirus disease 2019 (COVID-19) RNA positive by real-time polymerase chain reaction (PCR) by Baoan District People's Hospital. Shenzhen CDC received their oral nasopharyngeal swabs packages from the hospital and retested COVID-19 RNA positive at 09:50. Meanwhile, Case D, a close contact of Case A, was transferred to the designated hotel for centralized isolation for 14 days. His oral nasopharyngeal swabs were collected and sent for the detection of COVID19 RNA as routine surveillance testing on the 1, 4, 7, 14 days of quarantine; these were reported back as COVID-19 RNA positive at 22:50 on January 4, 2021 and at $05: 30$ on January 5, 2021 by Shenzhen CDC. The four cases were transferred respectively by negative pressure ambulances to the Third People's Hospital of Shenzhen for further treatment within five hours of COVID-19 infection confirmation by Shenzhen CDC. On admission, chest CT images showed no abnormalities among all cases.

Epidemiological investigation revealed that the 4 cases had COVID-19 RNA negative test results before departure and self-reportedly had effective protection by wearing medical protective masks (Case A wore a disposable medical mask, while Case $\mathrm{B}, \mathrm{C}$, and $\mathrm{D}$ wore N95 masks) and protective suits (Case B) during the flight. In addition, they reported no history of fever, fatigue, or respiratory symptoms within 14 days prior to their diagnosis. However, according to the report of Case A, he was possibly exposed on his return business trip from Cape Town to Johannesburg in South Africa from December 16 to December 30, 2020, when he visited and had dinner with colleagues and had a meal in a fast food restaurant. Case B had a history of performing work-related tasks, staying at the dormitory with workmates. His roommate, who took the same flight from South Africa to China, was diagnosed as an asymptomatic carrier of COVID-19 on January 1, 2021 in Shenzhen. Case C's potential exposure might have been a 4-hour-trip in a vehicle from his company in Lesotho to an airport hotel with a local resident without effective protection. Case D's potential exposure was the experience that he took nucleic acid testing in South Africa and shuttle bus from workplace to the airport with Case A before boarding. Using the results of the epidemiological investigation, clinical symptoms, and laboratory results, these patients were diagnosed as asymptomatic patients with COVID-19 in coordination with clinicians and experts.

The specimens of the 4 asymptomatic patients' were sequenced by Illumina MiSeq Sequencing platform between January 2 to January 5, 2021. On January 4 and January 7, 3 whole genome sequences and 1 were obtained, respectively. Compared with the Wuhan reference sequence (EPI_ISL_402119) (1-2), these 4 newly identified Shenzhen strains showed 29 nucleotide variation sites, which contained the single nucleotide polymorphisms (SNPs) that defined lineage B.1.351 (20H/501Y.V2 branch) (3). A maximum of 29 genomic variation sites were detected in the 4 highquality genomic sequences (for Cases A-D, number of genomic variation sites were $29,19,25$, and 25 , respectively, including G160A, G174T, C241T, C281T, C1059T, C3037T, G5230T, C8660T, C8964T, A10323G, G11230T, T13324A, C14408T, C14925T, T16062C, G19656T, A21801C, G22299T, G22813T, G23012A, A23063T, A23403G, C23664T, T25084C, G25563T, C25904T, C26456T, C28647T, C28887T; sequence coverage ranged from $91.4 \%$ to $99.2 \%$ ). A major difference between the nucleotide sequences of $\mathrm{A} / \mathrm{D}$ 
and $\mathrm{B} / \mathrm{C}$ were detected in the position of 1174 (A1174C for $\mathrm{A} / \mathrm{D}$ and $\mathrm{A} 1174 \mathrm{~T}$ for $\mathrm{B} / \mathrm{C}$ ). By online comparison of the assembled sequence with the published COVID-19 database, we obtained the phylogenetic relationship tree (Figure 1) of the virus strains. The 4 COVID-19 samples of imported African cases were lineage B.1.351 (20H/501Y.V2) variant strains (homology of $99.99 \%$ to $100 \%$ ), which had been circulating in South Africa since October 2020 (4). The cladogram showed that the genome sequences of the 4 cases imported from Africa (Case A-D) all contained the characteristic mutation sites (the mutations of the spike protein D80A, K417N, E484K, N501Y, D614G, and A701V) of the South African variant strain B.1.351. The major differences between the $\mathrm{A} / \mathrm{D}$ and $\mathrm{B} / \mathrm{C}$ when comparing their amino acid sequences were as follows: $\mathrm{L} 18 \mathrm{~F}$ was detected in Case C; D215G was detected in Case B/C; R246I was detected in Case A; but data on the same position was missing in Case D. These B.1.351 variants strains were imported early to China and might exert a great threat to the prevention and control of COVID-19 in China. Systematically tracking higher risk international variants is therefore important as it allows monitoring of the new variants nationally and internationally.

Acknowledgements: Prof. Biao Kan and Prof. Xin Lu from China CDC; Prof. Yujun Cui and Hang Fan, $\mathrm{PhD}$ from Beijing Institute of Microbiology and Epidemiology; Prof. Jing Lu, PhD, from Guangdong Institute of Public Health; and patients who consented to donate their data for analysis.

Funding: Non-profit Central Research Institute Fund of Chinese Academy of Medical Sciences
(NO.2020-PT330-006), Shenzhen Key Medical Discipline Construction Fund (NO. SZXK064), and Sanming Project of Medicine in Shenzhen (NO. SZSM 201811071) and the China National Science and Technology Major Projects Foundation (NO.2017ZX10303406).

doi: $10.46234 / \mathrm{ccdcw} 2021.049$

\# Corresponding authors: Qinghua Hu, huqinghua03@163.com; Shujiang Mei, sjmei66@163.com; Tiejian Feng, fengtiej@126.com.

\begin{abstract}
${ }^{1}$ Department of Communicable Diseases Control and Prevention, Shenzhen Center for Disease Control and Prevention, Shenzhen, Guangdong, China; ${ }^{2}$ Microbiology Laboratory, Shenzhen Center for Disease Control and Prevention, Shenzhen, Guangdong, China; ${ }^{3}$ Key Laboratory of Molecular Epidemiology of Shenzhen, Shenzhen Center for Disease Control and Prevention, Shenzhen, Guangdong, China; ${ }^{4}$ Institute of pathogenic biology, Shenzhen Center for Disease Control and Prevention, Shenzhen, Guangdong, China; ${ }^{5}$ Shenzhen Center for Disease Control and Prevention, Shenzhen, Guangdong, China.

\& Joint first authors.
\end{abstract}

Submitted: January 21, 2021; Accepted: February 04, 2021

\section{REFERENCES}

1. Tan WJ, Zhao X, Ma XJ, Wang WL, Niu PH, Xu WB, et al. A novel coronavirus genome identified in a cluster of pneumonia cases Wuhan, China 2019-2020. China CDC Wkly 2020;2(4):61-2. http://dx.doi.org/10.46234/ccdcw2020.017.

2. Zhu N, Zhang DY, Wang WL, Li XW, Yang B, Song JD, et al. A novel coronavirus from patients with pneumonia in China, 2019. N Engl J Med 2020;382(8):727 - 33. http://dx.doi.org/10.1056/NEJMoa2001017.

3. Rambaut A, Holmes EC, O’Toole á, Hill V, McCrone JT, Ruis C, et al. A dynamic nomenclature proposal for SARS-CoV-2 lineages to assist genomic epidemiology. Nat Microbiol 2020;5(11):1403-7. http://dx.doi.org/10.1038/s41564-020-0770-5.

4. Tegally H, Wilkinson E, Giovanetti M, Iranzadeh A, Fonseca V, Giandhari J, et al. Emergence and rapid spread of a new severe acute respiratory syndrome-related coronavirus 2 (SARS-CoV-2) lineage with multiple spike mutations in South Africa. medRxiv. 2020. http://dx.doi.org/10.1101/2020.12.21.20248640v1. 


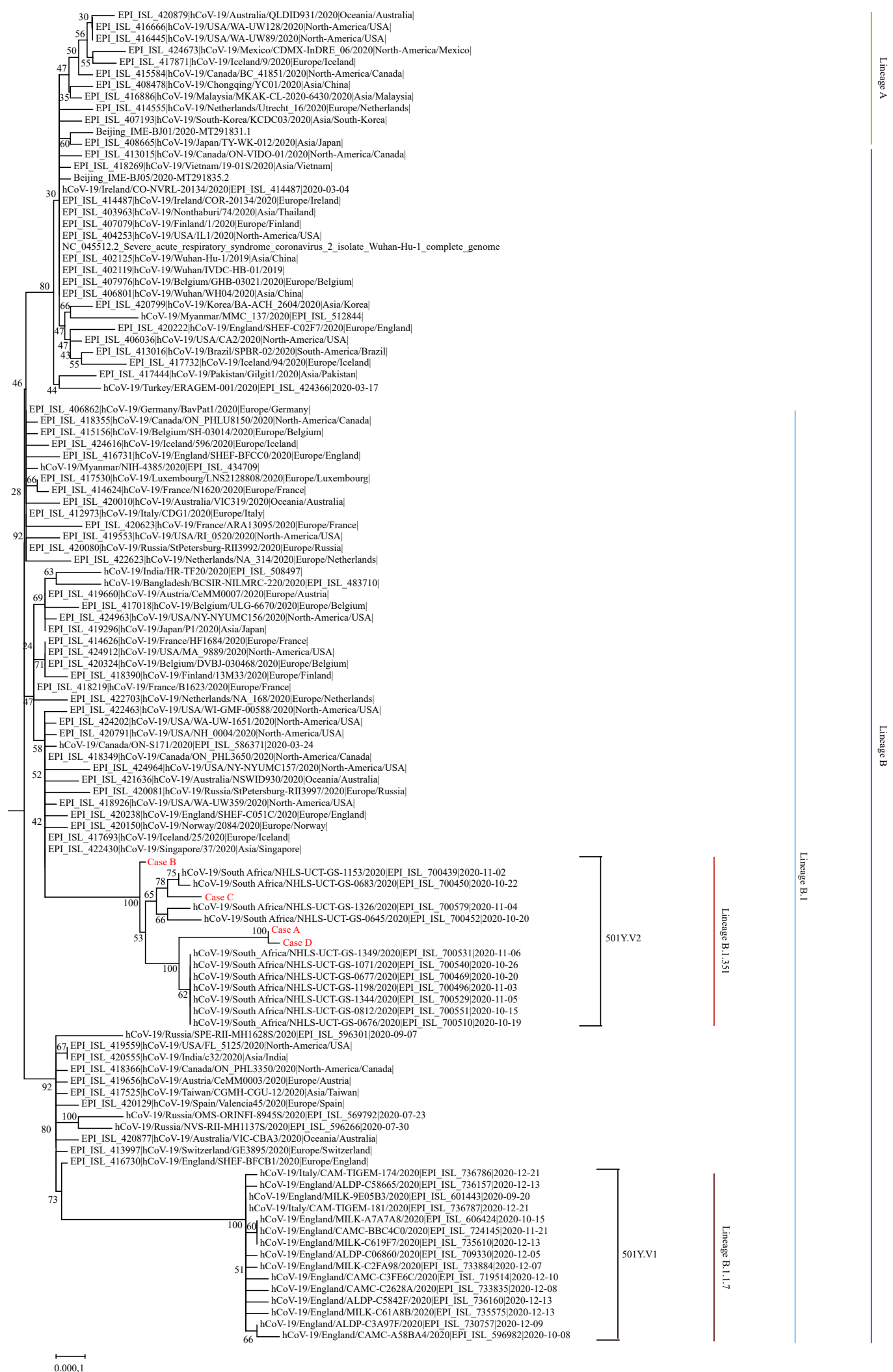

FIGURE 1. Phylogenetic tree based on the complete genome sequences of severe acute respiratory syndrome coronavirus 2 virus.

The four imported 501Y.V2 cases are colored in red letters. Scale bar indicates nucleotide substitutions per site. The lineages and sublineages of COVID-19 virus are marked and colored on the right. 